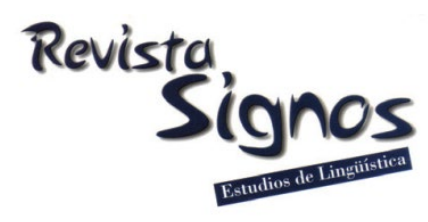

\title{
Narrativa autobiográfica y reminiscencias en costarricenses con Alzheimer
}

\author{
Autobiographical narrative and reminiscences in Costa Ricans \\ with Alzheimer
}

\section{Mónica Salazar-Villanea}

UNIVERSIDAD DE COSTA RICA COSTA RicA

monica.salazarvillanea@ucr.ac.cr

Recibido: 17-XI-2018 / Aceptado: 01-VII-2019

DOI: $10.4067 /$ S0718-09342020000100219

\section{Resumen}

La reminiscencia, como narrativa de la memoria autobiográfica, es una forma de intervención no farmacológica reconocida en el envejecimiento y el síndrome demencial. En este trabajo se busca comprender mejor la posible funcionalidad pragmático-discursiva de la reminiscencia en personas con Enfermedad de Alzheimer, preservando la interacción positiva en contextos socioculturales a través de actos comunicativos y brindando sentido de identidad. El objetivo fue caracterizar la estructura, el contenido y la construcción mnésico-discursiva de las narraciones autobiográficas ante la demanda comunicativa de una reminiscencia. Se realizó un análisis cualitativo de narrativas autobiográficas de 30 personas con Enfermedad de Alzheimer en fase inicial. Los resultados muestran narrativas autobiográficas poco específicas en cronología y completud de detalles, pero con una representación del 'sí mismo' que se asocia a un mantenimiento semántico de la identidad. Estudios previos han descrito hallazgos similares, como superestructuras discursivas, pues no cumplen con la calidad informativa que requiere la situación comunicativa. Frente a un creciente envejecimiento poblacional, el valor de esta posibilidad narrativa y comunicativa justifica la necesidad de continuar estudiando su potencial beneficio.

Palabras Clave: Reminiscencia, memoria autobiográfica, demencia, discurso, sí mismo. 


\begin{abstract}
Reminiscence, as an autobiographical memory narrative, is a well-known, nonpharmacological intervention for aging and dementia. In this study, we seek to better understand the possible pragmatic and discursive functionality of reminiscence in people with Alzheimer's disease, preserving positive sociocultural interactions through communicative acts and providing a sense of identity. The aim was to characterize the structure, content and mnesic-discursive construction of the autobiographical narratives in a communicative demand of reminiscence. A qualitative analysis of autobiographical narratives was performed with 30 Alzheimer's disease patients in their initial phase. Results show non-specific autobiographical narratives in chronology and completeness of details, but with a semantic self-representation associated with their identity maintenance. Previous studies have described similar findings as discursive superstructures, because they do not comply with the informative quality required by the communicative situation. Faced with an aging population, the value of this narrative intervention justifies continuing the study of its potential benefits.
\end{abstract}

Key Words: Reminiscence, autobiographical memory, dementia, speech, self.

\title{
INTRODUCCIÓN
}

La inversión de la pirámide poblacional, dado el envejecimiento demográfico, hace previsible el aumento del porcentaje de personas con deterioro cognitivo y afectación funcional y social, con datos que revelan la ocurrencia de un nuevo diagnóstico de demencia cada tres segundos en el mundo (Prince, Wimo, Guerchet, Ali, Wu \& Prina, 2015).

El síndrome demencial por Enfermedad de Alzheimer (EA), como una de las causas más frecuentes de demencia irreversible, se caracteriza por el deterioro de la memoria y otras funciones superiores como la atención, el lenguaje, el razonamiento y las funciones ejecutivas que afectan también la regulación de la emoción y del comportamiento (American Psychiatric Association (APA), 2013). En dicho deterioro progresivo, se altera significativamente el funcionamiento de la persona en sus roles culturales e interacciones ocupacionales y/o sociales (Jurado, Mataró \& Pueyo, 2013; Noggle \& Dean, 2015). Se requieren, por tanto, esfuerzos de investigación internacional novedosos que permitan trascender la descripción de la pérdida cognitiva y neuroconductual, para rescatar las habilidades preservadas que permitan la intervención a favor de mayor calidad de vida. En ese sentido, la preservación de elementos de la identidad personal en actos comunicativos con referentes autobiográficos resulta fundamental en este trabajo. Los estudios previos describen cómo las personas con demencia se esfuerzan por tratar de equilibrar el ajuste a los cambios en la vida causados por la enfermedad y el mantenimiento de su identidad (Karlsson, Savenstedt, Axelsson \& Zingmark, 2014).

Eustache, Laisney, Juskenaite, Letortu, Platel, Eustache y Desgranges (2013) afirman que el sentido de identidad se encuentra parcialmente preservado incluso en 
las fases avanzadas de la demencia tipo Alzheimer. Señalan que las descripciones sobre sí mismos de personas con EA se mantienen consistentes con la intencionalidad de conservar un sentido de identidad, a pesar de su déficit para actualizar el conocimiento sobre sus experiencias recientes.

En un sentido amplio, los estudios sobre intervenciones no farmacológicas resaltan los beneficios de estrategias de comunicación con reminiscencias, que estimulen la memoria autobiográfica pues, neuropsicológicamente, se preserva la capacidad de reconstruir recuerdos personales emocionalmente significativos y comunicarlos con intencionalidad, aún en etapas avanzadas en la enfermedad de Alzheimer (Clare \& Woods, 2001; Woods, Spector, Jones, Orrell \& Davies, 2005; Woods, Aguirre, Spector \& Orrell, 2012).

Los resultados de investigaciones internacionales documentan que tanto los pacientes, los familiares, así como los cuidadores informales y formales de personas con EA, refieren que la intervención con memoria autobiográfica y emoción es la que brinda mayores beneficios en el cuido centrado en la persona, preservando la interacción y comunicación positiva en contextos socioculturales y aportando sentido de identidad (Harmer \& Orrell, 2008; Spector, Gardner \& Orrell, 2011; Karlsson et al., 2014).

En esa línea, se han desarrollado en Costa Rica estudios previos de intervención con reminiscencia en personas con EA (Salazar-Villanea, 2010; Salazar-Villanea, Garnier- Villarreal, Johnson \& Montenegro-Montenegro, 2013; Salazar-Villanea, Liebmann, Garnier-Villarreal, Montenegro-Montenegro \& Johnson, 2015), analizando cuantitativamente la relación entre los componentes semánticos y episódicos de la memoria autobiográfica, constructos emocionales de afecto positivo - afecto negativo, funciones de memoria verbal y funciones ejecutivas. Sin embargo, en el presente trabajo interesó profundizar, exclusivamente desde una perspectiva cualitativa, lo singular y particular del caso en la comunicación de personas con EA al narrar una reminiscencia y, por tanto, la propuesta posee una perspectiva ideográfica. El objetivo fue caracterizar la estructura, el contenido y la construcción mnésico-discursiva de las narraciones autobiográficas ante la demanda comunicativa de una reminiscencia. Se ahondó en la comprensión de las características de las narraciones autobiográficas en la intención comunicativa de elaborar en el presente una reminiscencia, para analizar cualitativamente su posible funcionalidad discursiva, estructura, coherencia y valencia emocional.

En este artículo se presentarán las teorías que permiten comprender la posible funcionalidad pragmático-discursiva de la reminiscencia como parte de la memoria autobiográfica. Se planteará que surge de la interacción neuropsicológica de procesos representacionales y proposicionales en la memoria, la conciencia, el lenguaje, el razonamiento, la emoción y el funcionamiento ejecutivo, por lo que ha sido descrita 
teóricamente como una zona de convergencia de las perspectivas neurobiológicas, cognitivas, lingüísticas y culturales (Welzer \& Markowitsch, 2005; Markowitsch \& Welzer, 2010). Se especificarán los aspectos metodológicos y procedimentales del estudio realizado, así como el análisis cualitativo escogido y sus principales resultados.

\section{Marco teórico}

\subsection{Memoria autobiográfica y narrativa sobre el sí mismo}

Como lo planteaba Schacter (1996), el sentido del 'sí mismo' depende crucialmente de la experiencia subjetiva de recordar el pasado, por lo que los recuerdos autobiográficos emocionalmente significativos son los que forman el núcleo de la identidad personal, sea o no que esos recuerdos puedan narrarse. La narración autobiográfica, en cambio, implicaría pensamiento, imagen, simbolización y lenguaje combinados en un metanivel (Fivush \& Haden, 2003; Nelson \& Fivush, 2004), con un componente reflexivo y creador de sentido, que transforma el recuerdo de la experiencia y el entorno en una estructura neural organizadora del 'sí mismo' (Markowitsch \& Welzer, 2010).

Cuando la memoria autobiográfica se explicita en una narrativa autobiográfica, como lo propusieron Lebel y Beaulieu (2011), tiene como precondiciones un desarrollo cerebral dependiente de la experiencia y un fenómeno de cognición y comunicación biocultural pues, todo recuerdo autobiográfico emerge desde y en un contexto social con la intencionalidad de ser comunicado. En ese sentido, se ha propuesto que el ‘sí mismo' posee múltiples dimensiones, pero es en su historización y comunicación que se forma la identidad (Rubin, 1986; McAdams, 2003). Para organizar una narrativa sobre el 'sí mismo', surgida de la memoria autobiográfica, se requiere la capacidad de integración sincrónica del 'yo' que narra y el 'mi' de la vivencia, así como integración diacrónica atribuyendo significado y valor al tiempo y a los cambios en la vividez de la experiencia que se recuerda y se comparte (Sutin \& Robins, 2008; Janssen, Rubin \& St. Jacques, 2011). En la emergencia y comunicación de esa narrativa del 'sí mismo', cuya coherencia surge de la búsqueda de sentido temporal, biográfico, causal o temático, hay por tanto diferencias culturales (Wang, 2004). Sin embargo, sobre la intencionalidad de la memoria autobiográfica y su comunicación discursiva, se ha planteado que parece buscar siempre identidad en la sincronización psicosocial del 'yo' narrador y el 'mi' narrado, que, desde una perspectiva evolutiva, es crucial como acto autoreflexivo y función reguladora frente a quien escucha, para brindar puntos de referencia que orienten y direccionen en las fluctuaciones de la vida y los roles diversos en situaciones, tiempos y lugares distintos (Conway \& Pleydell- Pearce, 2000; Markowitsch \& Welzer, 2010; Fuentes \& Desrocher, 2012). 


\subsection{Semantización en la memoria autobiográfica e identidad}

Se ha propuesto que la identidad requiere de la creciente semantización del funcionamiento de la memoria autobiográfica durante el ciclo vital (Piolino, Desgranges \& Eustache, 2009), donde eventos personales recientes pueden tener más componentes episódicos en su reconstrucción, mientras que eventos remotos son reconstruidos con componentes más semánticos, llegando incluso a constituirse en esquemas o guiones de vida de momentos prototípicos, distintivos y emocionalmente significativos que resultan de interés para el 'sí mismo' o para otros (Berntsen \& Rubin, 2002, 2004; Janssen et al., 2011; Zaragoza-Scherman, Salgado, Shao \& Berntsen, 2017). Se ha demostrado incluso que la actividad neuroanatómica y neurofuncional de la memoria autobiográfica varía a lo largo del ciclo vital cuando se reconstruyen recuerdos recientes o lejanos en el tiempo (Svoboda, Mckinnona \& Levine, 2006; Markowitsch \& Wezler, 2010). Estos hallazgos sobre los diversos tipos de reconstrucciones mnésicas autobiográficas que implican sustratos neuropsicológicos disímiles, cuestionan las variadas taxonomías para la comprensión de los procesos de memoria a largo plazo (Tulving \& Markowitsch, 1998) y permiten un replanteamiento de la distinción binaria entre memoria episódica y memoria semántica como tipos de memoria declarativa que los estudios clásicos, con materiales verbales neutros en tareas experimentales, no podrían haber dilucidado.

Suponen también la coexistencia en la memoria autobiográfica de los llamados 'tipos de conciencia' implicados en cualquier acto discursivo: la conciencia autonoética, que refiere al 'sí mismo' que re-experimenta el acontecimiento del pasado (reconstrucción entendida por mucho tiempo como memoria episódica en tanto situada en tiempo y espacio) y la conciencia noética, que parece remitir a un conocimiento básico personal, de tipo semántico, sin la experiencia subjetiva de revivir el pasado al comunicar (Piolino et al., 2009).

Se propone entonces que la memoria autobiográfica y las narrativas del 'sí mismo' que emergen y se comunican, podrían dar cuenta de acontecimientos vitales significativos y específicos, distintivos por su carga emocional (Thompson, Herrmann, Bruce, Read, Payne \& Toglia, 1998; Philippot \& Schaefer, 2005; Schulkind \& Woldorf, 2005; Holland \& Kensinger, 2010); pero también podrían dar cuenta de conocimiento personal, representacional y proposicional. Ambos poseen un valor de veracidad y, en su interconectividad, permiten el surgimiento de narrativas sobre el 'sí mismo' con organización temática y temporal, así como procesos de esquematización en los actos discursivos. Los diferentes sistemas cerebrales se activan de una manera paralela para representar experiencias en memoria episódica con detalles sensoriales-perceptivoscognitivos-afectivos que brindan especificidad al recuerdo, así como para representar esquemas, guiones y significados asociados al 'sí mismo' en memoria semántica. Los estudios muestran evidencia neuropsicológica de que el componente episódico parece 
deteriorarse con la edad y el intervalo de retención, privilegiando componentes semánticos a largo plazo (Hou, Miller \& Kramer, 2005; Burianova, Mcintosh \& Grady, 2010; Piolino et al., 2009; Hongkeun, 2012; Addis, Knapp, Roberts \& Schacter, 2012).

Esta comprensión de la memoria autobiográfica es similar a la que Kasabova (2009) proponía como necesaria para permitir encuentros entre las teorías filosóficas tradicionales sobre la memoria (como las de Bernard Bolzano, Ewald Hering y la fenomenología de Edmund Husserl) y las teorías derivadas de las ciencias cognoscitivas y neurociencias contemporáneas. Reconoce una estructura jerárquica, con organización semántica, representacional y enmarcada en la noción de 'tiempo' (marco temporal de referencia), que sigue reglas en la posible combinación de episodios por ser narrados. Las condiciones, señala Kasabova (2009), son un sentido de identidad en la sensación de conexión con el 'sí mismo semántico', gracias a la capacidad de viajar mentalmente en el tiempo personal como contexto de referencia; por lo que la memoria autobiográfica es, a la vez, 'construcción de conocimiento' y 'fuente de conocimiento' en la 'capacidad mental' que implica una representación renovada y un rol reflexivo (evento pasado con reconstrucción presente, que en fenomenología se ha llamado 'presentificacion').

\subsection{Acción comunicativa e identidad}

En la comprensión de la memoria autobiográfica tal y como aquí se ha planteado, se evidencia también la visión del recuerdo autobiográfico en la dimensión pragmática del lenguaje como acción comunicativa y supone un acto reflexivo del análisis o razonamiento biográfico (Habermas, 2011), que da sentido de coherencia y preserva la identidad (Libby \& Eibach, 2002).

En esa línea, interesa reconocer en este estudio cómo se estructura cualitativamente esa narrativa autobiográfica en condiciones de alteración del funcionamiento neuropsicológico, describiendo los procesos de semantización que preservan las referencias de identidad; a pesar de las alteraciones en la memoria, el lenguaje y el razonamiento esperables en la Enfermedad de Alzheimer y otras demencias (Sadek, Johnson, White, Salmon, Taylor, Delapena, Paulsen, Heaton \& Grant, 2004; Jetten, Haslam, Pugliese, Tonks \& Haslam, 2010; Duvala, Desgrangesa, De la Sayette, Belliard, Eustachea \& Piolino, 2012; Hydén, Lindemann \& Brockmeier, 2014; Karlsson et al., 2014; El Haj \& Antoine, 2017).

Por tanto, en este trabajo, se busca ampliar las comprensiones tradicionales del análisis del discurso en las prácticas del lenguaje, para incorporar los significados e interpretaciones discursivas e identitarias de la persona que habla, interesados en el cuándo, cómo y dónde de su producción, en lo que algunos teóricos han llamado el discurso en acto, en su dimensión más pragmática, semántica e histórica asociada a la subjetivación (Orlandi, 2014; Fontanille, 2016). El discurso de la reminiscencia de una 
persona con Enfermedad de Alzheimer, se entiende entonces en este trabajo, como forma de producción de sentido sobre la propia historia de vida y como referente de su identidad frente a una persona interlocutora que demanda la construcción de dicha narración, o sea, en situación de comunicación, más allá de las capacidades conservadas o alteradas del habla o el lenguaje propias de la enfermedad neurodegenerativa. Interesará metodológicamente una aproximación al análisis del discurso tanto en su forma como en su contenido (Orlandi, 2014), tal y como se especificará en el diseño de la investigación.

\section{Marco metodológico}

Si bien los análisis de discurso son reconocidos como un recurso valioso para el estudio de las dificultades de lenguaje en personas con EA (Lewis, 2016), los antecedentes metodológicos de investigaciones específicas, asociadas con el tema de memoria autobiográfica o preservación de la identidad en la EA, son muy limitados. Análisis del discurso con narrativas de personas con EA a nivel macrolingüístico, destacan el deterioro en la emisión de información relevante en las unidades de contenido, con características como discurso menos informativo, con mayor deterioro en la coherencia global, mayor modalización y estructura narrativa inferior (Toledo, Aluísio, Borges, Dozzi, Sturzeneker, Okada \& Lessa, 2017).

El trabajo que aquí se presenta, tal y como se detallará en el apartado de diseño metodológico, es transversal y descriptivo, con base en un análisis secundario y cualitativo de narraciones derivadas de estudios previos sobre reminiscencia en personas con EA y con el protocolo adaptado para Costa Rica (Salazar-Villanea, 2010; Salazar-Villanea et al., 2013; Salazar-Villanea et al., 2015). En las investigaciones previas, se analizó cuantitativamente la relación entre los componentes semánticos y episódicos de la memoria autobiográfica, constructos emocionales de afecto positivo afecto negativo, funciones de memoria verbal y funciones ejecutivas. En este artículo, interesó rescatar exclusivamente la perspectiva cualitativa e ideográfica, describiendo lo singular y particular de narraciones de personas con EA al comunicar una reminiscencia.

Se realizó un análisis de contenido de las narrativas autobiográficas surgidas en contextos de reminiscencia, siguiendo el protocolo estructurado, adaptado y validado para población costarricense (Salazar-Villanea, 2010).

\subsection{Participantes}

La muestra incluyó las narraciones autobiográficas de 30 personas con diagnóstico clínico de EA en fase inicial, con una media de edad de 70.50 años $(\mathrm{DE}=8.41)$, $60,08 \%$ mujeres. Su promedio de escolaridad fue de 11.56 años de educación (SD = 5.1), lo que en Costa Rica corresponde, en promedio, a la secundaria completa (sin 
estudios universitarios en su mayoría). Todas las personas residían en zonas urbanas del Valle Central de Costa Rica y tenían como idioma materno el español. Fueron contactadas a través de la Asociación Costarricense de Alzheimer y Otras Demencias Asociadas (ASCADA), la Fundación Alzheimer Costa Rica (FundAlzheimer), la Federación Cruzada Nacional de Protección al Anciano (FECRUNAPA), residencias y centros de día, entre otros. Su fase leve de EA, según su nivel de deterioro cognitivo, fue medido por el test MMSE en el cumplimiento de criterios de inclusión y oscilaba entre 15 y 24 puntos, con un Global Deterioration Scale for Assessment of Primary Degenerative Dementia (GDS de Reisberg) 3 - 4: presencia de déficits incipientes del funcionamiento cognitivo y conductas relacionadas con la Enfermedad de Alzheimer leve.

Estas 30 personas corresponden a una submuestra que cumplía con los criterios de un diagnóstico clínico de Enfermedad de Alzheimer, con deterioro cognitivo leve o moderado, de un protocolo de investigación más amplio con personas adultas mayores. La muestra total de participantes adultos mayores de dicha investigación, de la que este análisis se deriva, fue de 184 personas y su objetivo fue comprender cómo la salud mental y la salud cognitiva se relacionan en el envejecimiento. Los resultados cuantitativos de la investigación más amplia ya han sido publicados utilizando modelos de ecuaciones estructurales y análisis factoriales confirmatorios, que permiten establecer relaciones entre variables cognitivas, socioemocionales y físicas (SalazarVillanea et al., 2015).

Los 30 participantes fueron reclutados durante un período de 2 años de manera voluntaria siguiendo los criterios de inclusión y exclusión que, a continuación, se detallan en el Cuadro 1: 
Cuadro 1. Criterios de inclusión y exclusión.

\begin{tabular}{|c|c|}
\hline Criterios de inclusión & Criterios de exclusión \\
\hline $\begin{array}{l}\text { - Participante y familiar deben haber recibido la } \\
\text { información del estudio y estar dispuestos a } \\
\text { continuar en el proceso de intervención con } \\
\text { reminiscencia durante los siguientes } 2 \text { meses } \\
\text { después de firmar su consentimiento informado } \\
\text { para participar (o firma de tutor legal). } \\
\text { - Tener como lengua materna el español. } \\
\text { - Personas mayores de } 60 \text { años con diagnóstico } \\
\text { clínico de Enfermedad de Alzheimer } \\
\text { posible/probable brindado previamente por un } \\
\text { especialista en geriatría, que cumpla con los } \\
\text { criterios de la Clasificación Internacional de } \\
\text { Enfermedades (CIE-10) de la Organización } \\
\text { Panamericana de la Salud y Organización Mundial } \\
\text { de la Salud, los criterios del Manual diagnóstico y } \\
\text { estadístico de los trastornos mentales de la } \\
\text { Asociación Estadounidense de Psiquiatría (DSM- } \\
\text { IV). } \\
\text { - Con un GDS } 3 \text { - } 4 \text { en su sospecha diagnóstica } \\
\text { previamente establecida por el especialista en } \\
\text { medicina (Escala de Deterioro Global de Reisberg } \\
\text { donde } 3 \text { corresponde a deterioro cognitivo leve y } 4 \\
\text { corresponde a deterioro cognitivo moderado). } \\
\text { - Con deterioro cognitivo leve - moderado al } \\
\text { aplicarse el MiniMental State Examination en su } \\
\text { versión adaptada para Costa Rica (MMSE <24 y } \\
>15 \text { ). } \\
\text { - Contar con el apoyo y disposición de un familiar } \\
\text { para colaborar durante el proceso. }\end{array}$ & $\begin{array}{l}\text { - Personas que presenten patología psiquiátrica, } \\
\text { neurológica y/o neuropsicológica previa o asociada } \\
\text { (diferente al cuadro de deterioro cognitivo } \\
\text { asociado a la demencia por Enfermedad de } \\
\text { Alzheimer), que pueda interferir en el proceso. Por } \\
\text { ejemplo, tumor cerebral, hidrocefalia } \\
\text { normotensiva, endocrinopatías, patología sistémica } \\
\text { grave, epilepsia, traumatismo craneoencefálico, } \\
\text { eventos cerebrovasculares. } \\
\text { - Presencia de otros diagnósticos de demencia. } \\
\text { - Presencia de alteraciones graves en los procesos } \\
\text { de producción y comprensión del lenguaje que } \\
\text { impidan la comunicación e interacción oral. } \\
\text { - Presencia de deterioros graves de la visión o la } \\
\text { audición. } \\
\text { - No contar con el apoyo y disposición de un } \\
\text { familiar para colaborar en el proceso. } \\
\text { - Presencia de trastornos graves de conducta. }\end{array}$ \\
\hline
\end{tabular}

Para valorar que el nivel del deterioro cognitivo se mantuviera en leve o moderado en correspondencia con una fase inicial de EA, se utilizó el MiniMental State Examination (MMSE) como un instrumento de cribado que se utiliza en síndromes demenciales para medir el deterioro cognitivo en 5 dimensiones: orientación, memoria inmediata, atención y cálculo, lenguaje y praxis constructiva. La prueba tiene un puntaje total de 30 puntos con preguntas que evalúan el rendimiento en orientación temporal y espacial, memoria inmediata con la repetición de 3 elementos, atención y cálculo con una resta mental progresiva (100 menos 7), recuerdo de los 3 elementos, lenguaje con denominación, comprensión y seguimiento de instrucciones, escritura y lectura y, finalmente, praxis constructiva con una copia de un dibujo. La calificación se obtiene sumando los puntajes asignados en cada pregunta y se interpreta que altas puntuaciones indican un mayor rendimiento en las funciones evaluadas. Se utilizó la versión adaptada para Costa Rica (Castro-Rojas \& Salazar-Villanea, 2014). 


\subsection{Diseño de la investigación}

El diseño de investigación fue transversal y de alcance descriptivo, pues se recolectaron datos en un tiempo único, sin reevaluaciones ni seguimiento longitudinal y se procedió a recolectar los datos con el interés de explorar y describir el fenómeno cualitativamente, sin hipótesis de causalidad con otras variables independientes ni intereses de control o manipulación experimental de variables en la construcción de los relatos.

Se respetaron todos los criterios éticos y legales para la protección de las personas participantes y las transcripciones se anonimizaron para su análisis cualitativo, eliminando las referencias que pudiesen permitir la identificación de cada persona. Se trabajó con una muestra intencional, dada la perspectiva ideográfica del estudio, que buscó captar el contenido comunicativo manifiesto del texto, analizando las categorías de estructura, función y contenido de las reminiscencias, así como su valencia emocional, según el protocolo utilizado y validado para Costa Rica, tal y como se muestra en la Figura 1 (Salazar-Villanea, 2010).
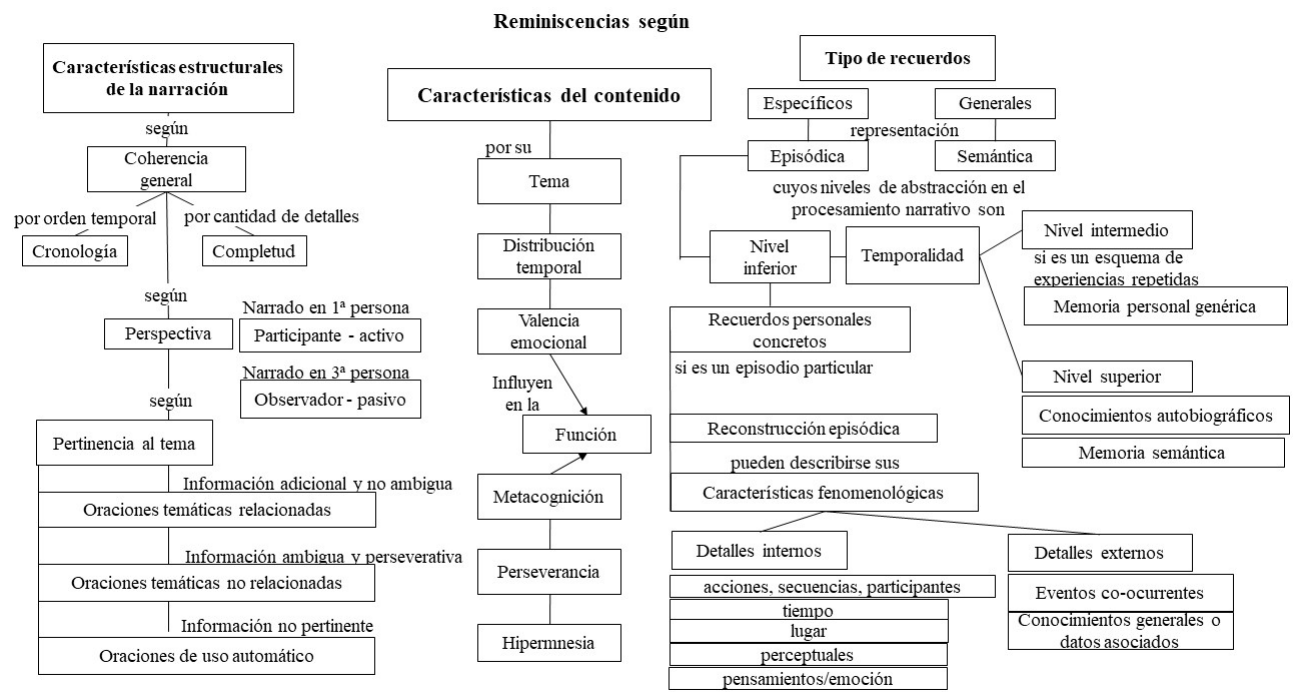

Figura 1. Sistema de clasificación de reminiscencias según estructura, función y contenido

\subsection{Materiales y procedimiento}

Las 30 personas participantes, en recolecciones de datos individuales de 30-50 minutos, reconstruían reminiscencias específicas con aproximación temática, utilizando claves o estímulos verbales y visuales homogéneos para la narración de recuerdos asociados a 6 dominios básicos: vida en el hogar; amor y relaciones de pareja; festividades, fiestas y días especiales; ocio, tiempo libre, juegos y amistad; época escolar y formación; trabajo. Los estímulos visuales que se presentaban como claves 
incluyeron fotografías e imágenes validadas para Costa Rica, todas ellas situacionales, pues conjugaban personas y acciones en escenarios concretos. Para definir el nivel de demanda cognitivo-lingüística en la tarea de reminiscencia, se consideraron como pautas básicas las siguientes: el grado de abstracción-concreción en el estímulo; los procesos metacognitivos implicados y el uso de claves progresivas.

Así, por ejemplo, tal y como se establece en las pautas de Salazar-Villanea (2010), en la demanda cognitiva superior, el estímulo que se utiliza es un estímulo verbal general, con preguntas abiertas sobre un tema clave que demanda mayores recursos de control ejecutivo para la abstracción y búsqueda estratégica de la información autobiográfica en la memoria a largo plazo. Supone un nivel expresivo-discursivo con la habilidad preservada de una reconstrucción narrativa estructurada y con sentido de recuerdo episódico. A este nivel de demanda corresponderían también las reflexiones metacognitivas en el discurso sobre los significados de la experiencia para el pasado y el presente personal. Cuando la persona presenta un deterioro cognitivo mayor, se estructura el uso progresivo de claves verbales cada vez más concretas a las que se asocian posteriormente preguntas específicas y claves visuales. Aunque las habilidades lingüísticas y expresivas se mantienen, es esperable que se requieran de estas claves adicionales cuando el relato es más desorganizado e incluso puedan existir ciertos errores de denominación o bien asociaciones discursivas atípicas o no relacionadas con el tema. Salazar-Villanea (2010) señala inclusive que, si la expresión verbal se limita a frases simples o palabras únicas, deberán brindarse preguntas y claves aún más concretas asociadas al tema.

Para transcribir los audios de las grabaciones con narraciones orales al formato de texto escrito, se consideraron las guías de transcripción y programación en el software Atlas-ti versión 6.2, con las siguientes pautas y códigos de transcripción para cada texto: las palabras, frases y oraciones se transcriben literalmente, sin alterar el discurso de la persona y mostrando fielmente incluso los errores, palabras inacabadas o repetidas, las llamadas 'muletillas' o expresiones dubitativas o ambiguas (ehh, diay...), usando además claves de signos como (?) para entonación interrogativa, (!) para entonación admirativa, (,) para una pausa menor de 2 segundos, (..) para una pausa de 2 a 5 segundos, (...) para una pausa de 5 a 10 segundos, (Tose) (Sonríe) los elementos no verbales se describen entre paréntesis.

En el software de análisis cualitativo, se codificaron las narrativas según las categorías nominales preestablecidas para la estructura, función y contenido de las reminiscencias, así como su valencia emocional, según el protocolo validado para Costa Rica y mostrado previamente en la Figura 1 (Salazar-Villanea, 2010). Las respuestas sí/no ante claves no se consideraban narrativas de reminiscencias codificables. 
Las unidades de registro para organizar y analizar los datos incluyeron palabras, oraciones o párrafos, en los que se identificaban también los períodos vitales a los que se hace referencia: niñez, adolescencia, adultez temprana entendida para este estudio como anterior a los 35 años, adultez media, que para efectos de este estudio se entiende como el período comprendido entre los 35 y los 65 años y adultez mayor o vejez, que para efectos de este estudio se entiende como el período vital posterior a los 65 años.

Se identificaban además las referencias o reflexiones sobre la valencia emocional (positiva, negativa o ambivalente) y percepciones asociados a esos relatos autobiográficos. Las lecturas múltiples del material textual en cada transcripción, buscaron que las categorías, en opinión de la persona investigadora y de otros jueces, reflejaran el contenido que encierran en su intención comunicativa.

Con el fin de garantizar la calidad de los datos, se buscó cumplir con el criterio de información adecuada que refiere a la cantidad de información recolectada y no al número de sujetos. Este criterio se logra cuando suficiente información ha sido recolectada al punto de que ocurre la saturación y se presenta la variación temática. En ese sentido, se trabajó con un total de 98 narraciones autobiográficas como unidad de análisis. Los datos estadísticos que se presentan en los resultados, corresponden al cálculo de los porcentajes de cuántas de las 98 narraciones autobiográficas analizadas presentan la característica a la que se hace referencia (categorías nominales preestablecidas de estructura, función y contenido). En ese sentido, se consideran las 98 narraciones como el universo completo de los datos, esto es, el 100\% y los diversos porcentajes que se presentan se han calculado en función de la frecuencia de codificación de la categoría nominal con el apoyo del software de análisis cualitativo Atlas-ti. Se consideraron también los criterios de credibilidad, transferibilidad y contrastación con jueces expertos del procedimiento y de las categorías de análisis surgidas del análisis de contenido descriptivo y cualitativo que fue desarrollado por la investigadora. Se calculó la concordancia interjueces de la codificación medida a partir del Kappa de Cohen. Para todas las categorías de análisis se lograron niveles de concordancia superiores a 0.81 .

\section{Resultados}

Sobre la coherencia general de las narrativas autobiográficas de personas con EA, debe decirse que la estructura narrativa mostró, en la mayoría de los casos (94\%), una carencia significativa de cronología y completud. Las narrativas construidas ante la demanda comunicativa de una reminiscencia, se caracterizaban por incorporar solamente detalles sobre el lugar y algunos participantes, en narrativas autobiográficas generales, con datos semánticos de contexto y conceptos o conocimientos asociados. Cuando involucraban componentes temporales, solían ser de nivel superior, con 
recuerdos referidos a períodos de vida o etapas duraderas que se pueden medir en años o décadas.

Solo un $9 \%$ de los relatos referían a episodios que pueden medirse en días, semanas o meses o niveles de mayor especificidad, que se miden en minutos y horas. Así, predominaron las narrativas clasificables como memorias personales genéricas, donde un 68\% estaban referidas a experiencias repetidas en esquemas o guiones de períodos de la historia de vida, con conocimientos autobiográficos globales. Este resultado, ha sido también descrito en otras investigaciones como una superestructura discursiva, que sigue un tema distinguible en su globalidad como una unidad completa, aunque no cumplen con la calidad informativa que requiere la situación comunicativa (Machado, Martínez, Figueroa \& Reyes, 2018).

Globalmente, las narrativas en todos los casos fueron pertinentes al estar temáticamente relacionadas con la consigna o demanda comunicativa, aunque mostraban alteraciones en la fluidez del lenguaje, anomia y parafasias o evidenciaban una carencia de detalles internos sobre la lógica secuencial de acciones, con mención de pocos aspectos perceptuales y pensamientos o emociones asociadas, tal y como se ejemplifica a continuación:

Ejemplo 1:

Participante: (..) trabajé en la casa y en la escuela

Entrevistadora: cuénteme

Participante: como maestra bueno tuve (,) un grupo de chiquillos bien malcriados

Entrevistadora: tuvo un grupo de chiquillos (,) muy malcriados (?)

Participante: (ríe) y uno quería que fueran bien educados (,) pero no era tan fácil (,) porque ya lo traían de la casa porque (,) había chiquillos muy buenos (,) y otros muy fregones

Entrevistadora: otros muy fregones (?)

Participante: sí (,) y no había maestro de música

Entrevistadora: ajá

Participante: sí (,) y y mamá (,) les enseñaba a los chicos (,) el ritmo de Perú y el himno de Panamá

Entrevistadora: su mamá les enseñaba qué (?)

Participante: el himno de Panamá y el de Perú

Entrevistadora: ah los ritmos (,) los himnos 
Participante: sí (..) y se hacía una asamblea muy bonita (..) yo había estudiado en Heredia en la Escuela Normal (,) yo (,) trabajé mucho

En este primer ejemplo, pueden observarse algunas alteraciones del lenguaje en las parafasias por sustitución, donde se reemplaza una palabra por otra, dada su relación en el campo semántico: ritmo e himno. La narrativa se mantiene coherente como memoria personal genérica, en un nivel de conocimientos autobiográficos generales, referidos a la experiencia repetida en el esquema o guión de sus labores y expectativas en el ámbito laboral como docente. Como ocurre también en el ejemplo 2, que a continuación se presenta, la reminiscencia corresponde a una superestructura discursiva sin calidad informativa ni detalles que permitan ahondar más en el acto de comunicación.

Ejemplo 2:

Participante: (...) digamos yo (..) yo me críe en una finca aquí en (,) en Desamparados y mi abuelo era el administrador (,) y si hay algo que me molestaba era que me levantaran en la madrugada a ir a coger café (risas) sí sí (,) era algo que (,) que a mí me molestaba ya (...) me acuerdo perfectamente digamos que (,) que para mí era (,) era (,) lo único me gustaba de (,) bueno siempre he sido vago pero diay (,) después resulté muy trabajador (,) pero lo único que me gustaba de tomar café (,) eh de coger café (,) era cuando la abuelita llegaba con (,) primero que todo yo no eh (,) a mi criaron mis abuelos

\section{Entrevistadora: ajá}

Participante: mi mamá trabajaba y (,) el papá (,) bueno fui de los (,) de los que (,) nunca tuve papá (,) y entonces (...) ehh (,) vez lo que te digo es que todo (???) y resulta que (..) entonces (,) lo único que me gustaba era cuando llegaba mi abuelita ehh (,) como a las ocho (,) nueve de la mañana con los gallos de (..) de torta (..) de torta de huevo

En este segundo ejemplo, se ilustra cómo el esquema de experiencias que se narra posee mayores referencias emocionales e identitarias con respecto a su crianza y sus vivencias de períodos de vida de la infancia o juventud, con mención de valoraciones globales sobre la continuidad o discontinuidad en la percepción de sí mismo ("siempre he sido vago pero diay (,) después resulté muy trabajador”). Temáticamente su reconstrucción narrativa está relacionada con la situación comunicativa, pero muestra alteraciones en la fluidez del lenguaje y asociaciones laxas en algunas frases, con pocos detalles internos sobre alguna lógica secuencial de acciones.

Con características similares de narrativa personal genérica e información mnésica semántica, sin elaboración específica en la cronología en el relato o en la completud de detalles, puede observarse en el ejemplo 3 cómo la perseveración y repeticiones en el 
lenguaje son comunes en la comunicación de narrativas autobiográficas de personas con EA:

Ejemplo 3:

Participante: (,) eh (,) yo (,) ehh (,) bueno eh (,) si me gustaba bailar y todo eso (,) no bailaba mucho pero diay algo le hacía verdad (,) y entonces (,) eh (,) viene un primo mío (,) que era en realidad éramos primos pero éramos como (,) éramos como hermanos más bien (,) andábamos juntos (,) salíamos juntos (,) y resulta que había un baile (,) con una (,) una orquesta de ahí (,) en aquellos tiempos famosa (,) yo no bailaba mucho pero (,) sí me gustaba mucho bailar

Como pudo apreciarse en los tres ejemplos anteriores, la perspectiva narrativa fue construida en primera persona, al igual que en un $89 \%$ de las 98 narraciones autobiográficas, donde los participantes utilizaban una voz activa 'yo' para dar cuenta de sí mismos y en ocasiones, como en los ejemplos que a continuación se ilustra, la intencionalidad narrativa permitía algunas reflexiones evaluativas o metacognitivas sobre los significados vitales subjetivos en la narración:

Ejemplo 4:

Participante: yo lo que recuerdo es (...) mi mamá (,) me (,) tuvo a mí (,) de 50 años (!) entonces (..) la hermana (..) menor (,) la que estaba antes (,) entre ellas tenía 21 años (..) entonces yo (,) me crié (,) muy sola (,) aunque tenía hermanos (,) pero todos eran mayores (..) entonces yo siempre (..) mi mamá (..) este (,) pues seguro ya cansada y eso (,) pues no (,) no me chineó (,) la verdad no (,) no (,) habían (..) éramos siete hermanos (,) entonces yo (,) pequeñita me iba para donde una vecina (,) porque (,) ella si me chineaba (,) y lo que yo le pedía (,) me lo daba (..) cuando yo llegaba a la casa (,) mi mamá pegaba (,) y me decía (,) por qué anda en la vecindad (?) pero yo di (,) estaba tan pequeña que no (,) no le (,) podía expresar que yo sentía soledad en la casa (..) nunca se lo dije (..) entonces (,) esa vecina me daba de comer (,) me daba confites (,) me chineaba (,) recuerdo lo que mamá me castigaba (!) porque yo buscaba cariño en otra parte (,) pero yo nunca se lo expresé

En este cuarto ejemplo, la acción comunicativa refleja un acto reflexivo de razonamiento biográfico, que permite entender a la persona que escucha, la manifestación y expresión de su percepción subjetiva de soledad y necesidad de mayor proximidad afectiva en el periodo de su vida infantil. Muestra, en ese sentido, capacidad preservada para comunicar el significado vital subjetivo de su propia narración autobiográfica, aún en las condiciones de alteración del funcionamiento neuropsicológico propia de la EA. 
Con identificación clara de la valencia emocional asociada a sus discursos narrativos autobiográficos, se presentas también los ejemplos 5 y 6 , donde puede observarse que, en el acto comunicativo, parece reconstruirse intersubjetivamente la percepción de valía personal. En ambos casos, ese razonamiento biográfico parece asociarse a la experiencia laboral de obtener una remuneración por su trabajo, así como al sentido de responsabilidad y superación como referente de su identidad masculina.

Ejemplo 5:

Participante: si no (,) tengo recuerdos así pero (..) específicamente (..) yo aprendí (,) soy zapatero (,) aprendí el oficio de zapatería (,) cuando tenía 11 años (,) ya yo trabajaba (..) de zapatero y (,) y recuerdo muy muy con mucha alegría (,) la primera semana que yo me gané seis colones (...) y se los di a mi mamá (...) a mí me marcó eso (,) fue importante para mi (,) eso y la (,) la (,) la educación (,) que tuve (,) recuerdo que para mí la escuela fue algo (,) precioso verdad (,) yo (,) yo tenía un ansia (,) por leer

Ejemplo 6:

Participante: desde muy chiquillo me dediqué a la sastrería (,) a hacer sólo pantalonero nada más (,) pero resulta ser que (,) ehh (,) yo lo que analizó es que yo siempre fui bendecido por Dios

Entrevistadora: ajá

Participante: porque siempre caí en buenas manos (,) siempre la gente me ayudó a superarme (,) ehh (.) tuve eso (..) apoyo (,) mucho apoyo de las personas (,) y y (,) tal vez era por (,) no sé (,) no quiero decir que por lástima (,) pero tal vez es que me miraban a mí (...) posiblemente ellos miraban la capacidad que yo podía (,) que podía tener (..) así (,) siendo pantalonero y yo siempre (,) yo siempre he dicho (,) y lo sigo diciendo (,) que uno nunca (,) tiene que olvidar de donde viene (..) porque (,) es una forma (,) no de vanagloriarse uno (..) de uno (..) sino de ver lo que son las raíces de uno

Los referentes autobiográficos de identidad del pasado que se expresan, parecen mostrar en la intencionalidad comunicativa de la reminiscencia un esfuerzo por sostener coherencia con el sí mismo y su autopresentación a través del relato.

En cuanto a la distribución temporal, como se pudo observar en los anteriores ejemplos, la mayoría de narrativas correspondían a periodos vitales tempranos. Específicamente de las 98 narraciones autobiográficas, un 36\% referían al momento vital de la infancia, un $33 \%$ a la adolescencia y un $21 \%$ a la adultez temprana, con mención a la adultez media solamente en un $8 \%$ y a la vejez en un $2 \%$ de las narraciones. 
La valencia emocional fue predominantemente positiva y en un $82 \%$ las narrativas cumplían una función integrativa con referencias al 'sí mismo' y con razonamiento biográfico, en historias emocionalmente significativas o con reconocimiento, en el acto comunicativo, de un rol social o cultural como se ilustra en estos extractos de los ejemplos 7 y 8 :

\section{Ejemplo 7:}

Participante: ha sido (,) una lucha dura (...) pero con buenos beneficios (...) en mi vida bueno (..) el trabajo (,) el trabajo me lo dió todo

Entrevistadora: el trabajo (?)

Participante: yo trabajé desde güila (,) y el trabajo me dio posición (,) me dioo (..) ehh (,) como le dijera yo (..) libertad económica (..) me diooo (..) cosas que yo nunca hubiera logrado (..) ehh (,) un nuevo yo (,) ya tengo (,) voy a cumplir (...) no sé (,) tengo años de haberme pensionado (..) y aún (,) ehhh (..) la gente me saluda (,) los que me conocieron

Ejemplo 8:

Participante: mi niñez fue muy linda (,) muy trabajada (..) muy pobre (..) me (,) me gusta recordarlo (,) porque (,) siento como (,) como que fue algo (..) como también alegre (,) porque iba con mi mamá (,) mi mamá a todos lados me acompañaba y (,) aún hoy (..) pero de otras maneras uno (,) uno la lleva con uno (,) y esa alegría se (,) se (,) se guarda

Se aprecia así una proposición narrativa de significancia vital y emocional, con referencia a vínculos y roles del pasado personal que sostienen la identidad en la semantización del relato y la intención comunicativa.

\section{CONCLUSIONES}

En las narrativas autobiográficas de las personas costarricenses con Enfermedad de Alzheimer analizadas, se identifican patrones de reconstrucción y comunicación similares a las del estudio doctoral previo realizado por Salazar-Villanea (2008) con población española. El análisis cualitativo rescata la perspectiva ideográfica y coincide en sus resultados con investigaciones previas que documentan una intencionalidad comunicativa de mantenimiento de la identidad, con un sentido del 'sí mismo' parcialmente preservado en la Enfermedad de Alzheimer (Eustache et al., 2013; Hydén et al., 2014; Karlsson et al., 2014; El Haj \& Antoine, 2017). Las características distintivas, descritas en los resultados, muestran la preservación de una capacidad de reconstrucción autobiográfica que, a pesar de ser poco específica en cronología o completud de detalles, refiere a una representación sobre el 'sí mismo' con base en la cual se hace una proposición de significado vital en el acto comunicativo. 
Los recuerdos poseen significancia emocional para las personas con EA, quienes logran estructurar, en la narrativa que comunican, un decir sobre sí mismos aún y cuando, por las características neurocognitivas de su enfermedad demencial, el recuerdo tienda a la semantización y parezca más un contorno o esquema de experiencias. Éstas, sin embargo, parecen dar cuenta de un contenido previamente elaborado con mayores detalles episódicos originales, que ya no logran ser reconstruidos o expresados y comunicados por completo. En muchas ocasiones, incluso, se narra una especie de conocimiento o saber sobre el 'sí mismo', como componente netamente semántico de la memoria autobiográfica, sin recuerdo específico que pueda revivirse al ser comunicado.

Estos hallazgos, descritos como superestructuras discursivas en otros estudios, parecen mostrar una dificultad en la adaptación ante la demanda discursiva que sostiene un tema distinguible en su globalidad, aunque no cumplen con la calidad informativa que requiere la situación comunicativa (Machado et al., 2018).

Así, parece existir un deterioro de la memoria autobiográfica en el nivel de eventos específicos (nivel inferior de componentes episódicos), con pérdida de asociación contextual y lógica comunicativa secuencial, pero con adquisición de características discursivas semánticas, basado en esquemas temáticos o temporales más abstractos que dan cuenta del 'sí mismo' con valencia emocional predominantemente positiva.

Aunque por la literatura científica previa pudiese esperarse la introducción de contenido irrelevante o elementos fuera de tema, dadas las dificultades semánticopragmáticas documentadas en la EA, las narrativas que emergen en este estudio, parecen favorecer un contexto que reafirma un sentido de identidad, a través de la expresión del 'sí mismo semántico' que, recordando el pasado como contexto de referencia, sirve de fuente de conocimiento personal en un presente comunicativo.

En ese sentido, si el nuevo conocimiento es difícil de aprehender -y mirar al pasado es posible gracias al sustrato neuropsicológico y cultural del 'decir sobre el sí mismo' que se preserva en la EA-, la narrativa autobiográfica puede entonces servir de resguardo temporal de la identidad y de conexión comunicativa con la alteridad en familia y comunidad. Se convierte entonces en una posible herramienta terapéutica al ser la reminiscencia una vía de intervención de aplicación logopédica y pragmáticodiscursiva en personas con Enfermedad de Alzheimer.

Nuevas tendencias clínicas de intervención en lenguaje, que retoman modelos socioconstructivistas, podrían explorar los beneficios de las reminiscencias como estimulación de lenguaje y comunicación en entornos naturales de interacción de las personas con EA.

Si bien estos resultados no pueden generalizarse, dado su alcance descriptivo limitado a una población muy particular, el tema resulta de interés frente a un 
creciente envejecimiento poblacional que demanda mayor investigación en temas como los síndromes demenciales, sus impactos y manejos terapéuticos. El valor de esta posibilidad narrativa y comunicativa plantea la necesidad de continuar estudiando su potencial, sin dejar de reconocer la importancia de la variabilidad interindividual o intersubjetiva, que aumenta a lo largo del ciclo vital y se expresa en una gran diversidad discursiva en la ontogenia.

\section{REFERENCIAS BIBLIOGRÁFICAS}

Addis, D., Knapp, K., Roberts, R. \& Schacter, D. (2012). Routes to the past: Neural substrates of direct and generative autobiographical memory retrieval. Neuroimage, 59, 2908-2922.

American Psychiatric Association (2013). Diagnostic and statistical manual of mental disorders (5th ed.). Washington: Washington, DC.

Berntsen, D. \& Rubin, D. (2002). Emotionally charged autobiographical memories across the life span: The recall of happy, sad, traumatic, and involuntary memories. Psychology and Aging, 17(4), 636-652.

Berntsen, D. \& Rubin, D. (2004). Cultural life scripts structure recall from autobiographical memory. Memory \& Cognition, 32(3), 427-442.

Burianova, H., Mcintosh, A. \& Grady, C. (2010). A common functional brain network for autobiographical, episodic, and semantic Memory retrieval. Neuroimage, 49, 865-874.

Castro-Rojas, M. D. \& Salazar-Villanea, M. (2014). Rendimiento cognitivo de personas mayores que participan en grupos organizados en la provincia de San José. Actualidades en Psicología, 28(116), 41-53.

Clare, L. \& Woods, R. T. (Eds.) (2001). A role for cognitive rehabilitation in dementia care. Neuropsychological Rehabilitation, Special Issue, 11(3-4), 193-196.

Conway, M. \& Pleydell-Pearce, C. (2000). The construction of autobiographical memories in the self-memory system. Psychological Review, 107(2), 261-288.

Duvala, C., Desgrangesa, B., De la Sayette, V., Belliard, S., Eustachea, F. \& Piolino, P. (2012). What happens to personal identity when semantic knowledge degrades? A study of the self and autobiographical memory in semantic dementia. Neuropsychologia, 50, 254-265.

El Haj, M. \& Antoine, P. (2017). Describe yourself to improve your autobiographical memory: A study in Alzheimer's disease. Cortex, 88, 165-172. 
Eustache. M. L., Laisney, M., Juskenaite, A., Letortu, O., Platel, H., Eustache, F. \& Desgranges. (2013). Sense of identity in advanced Alzheimer's dementia: A cognitive dissociation between sameness and selfhood? Consciousness and Cognition, 22(4), 1456-1467 [en línea]. Disponible en: https://doi.org/10.1016/j.concog.2013.09.009

Fivush, R. \& Haden, C. (2003). Autobiographical memory and construction on a narrative self. Developmental and cultural perspectives. Mahwah, N.J.: Lawrence Erlbaum.

Fontanille, J. (2016). Semiótica del discurso. Universidad de Lima: Fondo Editorial.

Fuentes, A. \& Desrocher, M. (2012). Autobiographical memory in emerging adulthood: Relationship with self-concept clarity. Journal of Adult Development, 19(1), 28-39.

Habermas, T. (2011). Autobiographical reasoning: Arguing and narrating from a biographical perspective. En T. Habermas (Ed.), The development of autobiographical reasoning in adolescence and beyond. New Directions for Child and Adolescent Development (pp. 1-17). San Francisco: Jossey-Bass.

Harmer, B. \& Orrell, M. (2008). What is meaningful activity for people with dementia living in care homes? A comparison of the views of older people with dementia, staff and family carers. Aging and Mental Health, 12(5), 548-558.

Holland, A. \& Kensinger, E. (2010). Emotion and autobiographical memory. Physics of Life Reviews, 7, 88-131.

Hongkeun, K. (2012). A dual-subsystem model of the brain's default network: Selfreferential processing, memory retrieval processes, and autobiographical memory retrieval. NeuroImage, 61(4), 966-977.

Hou, C., Miller, B. \& Kramer, J. (2005). Patterns of autobiographical memory loss in dementia. International Journal of Geriatric Psychiatry, 20, 809-815.

Hydén, L., Lindemann, H. \& Brockmeier, J. (Ed.). (2014). Beyond Loss. Dementia, Identity, Personhood. Nueva York: Oxford University Press.

Janssen, S., Rubin, D. \& St. Jacques, P. (2011). The temporal distribution of autobiographical memory: Changes in reliving and vividness over the life span do not explain the reminiscence bump. Memory \& Cognition, 39, 1-11.

Jetten, J., Haslam, C., Pugliese, C., Tonks, J. \& Haslam, A. (2010). Declining autobiographical memory and the loss of identity: Effects on well-being. Journal of Clinical and Experimental Neuropsychology, 32(4), 408-416.

Jurado, M., Mataró, M. \& Pueyo, R. (2013). Neuropsicología de las enfermedades neurodegenerativas. Madrid, España: Editorial Síntesis. 
Kasabova, A. (2009). On autobiographical memory. UK: Cambridge Scholars Publishing.

Karlsson, E., Savenstedt, S., Axelsson, K. \& Zingmark, K. (2014). Stories about life narrated by people with Alzheimer's disease. Journal of Advanced Nursing, 70(12), 2791-2799.

Lebel, C. \& Beaulieu, C. (2011). Longitudinal development of human brain wiring continues from childhood into adulthood. Journal of Neuroscience, 31(30), 10937.

Lewis, T. (2016). Critical review: Exploring the use of discourse analysis in the early identification of Alzheimer's disease. Ontario: University of Western Ontario, School of Communication Sciences and Disorders [en línea]. Disponible en: https://www.uwo.ca/fhs/lwm/teaching/EBP/2015_16/Lewis.pdf

Libby, L. \& Eibach, R. (2002). Looking back in time: self-concept change affects visual perspective in autobiographical memory. Journal of Personality and Social Psychology, 82(2), 167-179.

Machado, A. P., Martínez, C., Figueroa, D. \& Reyes, C. (2018). Análisis de la funcionalidad discursivo-pragmática en adultos mayores sanos y con demencia leve. Logos: Revista de Lingüistica, Filosofía y Literatura, 28(1), 192-205.

Markowitsch, H. \& Welzer, H. (2010). The development of autobiographical memory. UK: Psychology Press.

McAdams, D. (2003). Identity and the life story. En R. Fivush \& C. Haden. (Eds.), Autobiographical memory and construction on a narrative self. Developmental and cultural perspectives (pp.187-223). Mahwah, N.J.: Lawrence Erlbaum.

Nelson, K. \& Fivush, R. (2004). The emergence of autobiographical memory: A social cultural developmental theory. Psychological Review, 111(2), 486-511.

Noggle, C. \& Dean, R. (2015). The Neuropsychology of Cortical Dementias. Nueva York: Springer Publishing.

Orlandi, E. (2014). Análisis de discurso: Principios y procedimientos. Santiago, Chile: LOM Ediciones.

Philippot, P. \& Schaefer, A. (2005). Selective effects of emotion on the phenomenal characteristics of autobiographical memories. Memory, 13(2), 148-160.

Piolino, P., Desgranges, B. \& Eustache, F. (2009). Episodic autobiographical memories over the course of time: Cognitive, neuropsychological and neuroimaging findings. Neuropsychologia, 47, 2314-2329.

Prince, M., Wimo, A., Guerchet, M., Ali, C., Wu, Y. \& Prina, M. (2015). Informe Mundial sobre el Alz̧beimer 2015. Las consecuencias de la demencia: Análisis de 
prevalencia, incidencia, coste y tendencias. Alzheimer's Disease International (ADI), Londres [en línea]. Disponible https://www.alz.co.uk/research/worldalzheimerreport2015-summaryspanish.pdf

Rubin, D. C. (Ed.) (1986). Autobiographical memory. Nueva York: Cambridge University Press [en línea]. Disponible en: http://books.google.co.cr/books

Sadek, J., Johnson, S., White, D., Salmon, D., Taylor, K., Delapena, J., Paulsen, J., Heaton, R. \& Grant, I. (2004). Retrograde amnesia in dementia: Comparison of HIV-associated dementia, Alzheimer's disease, and Huntington's disease. Neuropsychology, 18(4), 692-699.

Salazar-Villanea, M. (2008). Estudio de las características de la memoria autobiográfica en la vejez: Reminiscencia en adultos mayores sanos y con demencia tipo Alzhbeimer. Tesis doctoral, Universidad de Salamanca, Salamanca, España [en línea]. Disponible en: https://gredos.usal.es/handle/10366/22523

Salazar-Villanea, M. (2010). Recordando Experiencias. Programa de intervención con reminiscencia para personas adultas mayores. Guía para terapeutas y $C D$ con estímulos visuales. San José: Instituto de Investigaciones Psicológicas, Universidad de Costa Rica.

Salazar-Villanea, M., Garnier-Villarreal, M., Johnson, D. K. \& MontenegroMontenegro, E. (2013). Autobiographical memory relates to emotion and cognition in a sample of Costa Rican older adults. Ponencia presentada en The International Conference on Aging in the Americas, Universidad de Texas, Austin, USA.

Salazar-Villanea, M., Liebmann, E., Garnier-Villarreal, M., Montenegro-Montenegro, E. \& Johnson, D. (2015). Depressive symptoms affect working memory in healthy older adult hispanics. Journal of Depression and Anxiety, 4(4), 204.

Schacter, D. (1996). Searching for memory: The brain, the mind and the past. Nueva York: Basic Books.

Schulkind, M. \& Woldorf, G. (2005). Emotional organization of autobiographical memory. Memory \& Cognition, 33(6), 1025-1035.

Spector, A., Gardner, C. \& Orrell, M. (2011). The impact of cognitive stimulation therapy groups on people with dementia: Views from participants, their careers and group facilitators. Aging and Mental Health, 15(8), 945-949.

Sutin, A. \& Robins, R. (2008). When the 'I' looks at the 'me': Autobiographical memory, visual perspective, and the self. Consciousness and Cognition, 17, 13861397. 
Svoboda, E., Mckinnona, M. \& Levine, B. (2006). The functional neuroanatomy of autobiographical memory: A meta-analysis. Neuropsychologia, 44, 2189-2208.

Thompson, C., Herrmann, D., Bruce, D., Read, J., Payne, D. \& Toglia, M. (Ed.). (1998). Autobiographical memory. Theoretical and applied perspectives. Nueva Jersey: Lawrence Erlbaum.

Toledo, C. M., Aluísio, S. M., Borges, L., Dozzi, S. M., Sturzeneker, E., Okada, M. \& Lessa, L. (2017). Analysis of macrolinguistic aspects of narratives from individuals with Alzheimer's disease, mild cognitive impairment, and no cognitive impairment. Alz̧eimers Dement (Amst), 19(10), 31-40.

Tulving, E. \& Markowitsch, H. (1998). Episodic and declarative memory: Role of the hippocampus. Hippocampus, 8, 198-204.

Wang, Q. (2004). The emergence of cultural self-constructs: Autobiographical memory and self-description in European, American and Chinese children. Developmental Psychology, 40(1), 3-15.

Welzer, H. \& Markowitsch, H. (2005). Towards a bio-psycho-social model of autobiographical memory. Memory, 13(1), 63-78.

Woods, B., Spector, A. E., Jones, C. A., Orrell, M. \& Davies, S. P. (2005). Reminiscence therapy for dementia. Cochrane Database of Systematic Reviews, Issue 2. Art. No.: CD001120.

Woods, B., Aguirre, E., Spector, A. E. \& Orrell, M. (2012). Cognitive stimulation to improve cognitive functioning in people with dementia. The Cochrane Database of Systematic Reviews. DOI: 10.1002/14651858.CD005562.pub2

Zaragoza-Scherman, A., Salgado, S., Shao, Z. \& Berntsen, D. (2017). Life script events and autobiographical memories of important life story events in Mexico, Greenland, China, and Denmark. Journal of Applied Research in Memory and Cognition, 6, 60-73. 